\title{
RR Lyrae Studies with Kepler
}

\author{
Katrien Kolenberg and KASC WG13 (RR Lyrae working group)
}

\begin{abstract}
To date, about 40 known RR Lyrae stars are located in the Kepler field. The outstandingly high-precision data of these stars are investigated within the RR Lyrae working group as part of the Kepler Asteroseismic Science Consortium (KASC). I present some of the most interesting results obtained with Kepler data of RR Lyrae stars, and their implications for our understanding of RR Lyrae stars and the still mysterious Blazhko effect.
\end{abstract}

\section{RR Lyrae Stars in the Kepler Field}

The Kepler satellite was launched on March 7, 2009, and since shortly thereafter it has been staring at the same field in the sky. As a tool to detect transits of Earth-sized planets around stars, it delivers light curves of unprecedented precision (Fig. 1) of a variety of targets [2], including variable and pulsating stars [5].

RR Lyrae stars come in different flavors, and in the Kepler field we find radial fundamental mode pulsators, the RRab stars, many of which are modulated [1], and several radial first overtone pulsators, RRc stars. At the time of writing, about 40 RR Lyrae stars are known in the Kepler field with mean Kepler magnitudes (wide passband between 4,300 and $9,000 \AA$ ) in the range $7.9 \leq K_{p} \leq 17.4$.

Whereas long $(\simeq 30$-min) cadence provides a good coverage of the RR Lyrae light curve, only short ( $\simeq 1$-min) cadence allows us to distinguish specific features in the light curves, such as a shoulder on the steep rising branch of the curve, and

\footnotetext{
K. Kolenberg $(\bowtie)$

Harvard-Smithsonian Center for Astrophysics, 60 Garden Street, Cambride MA 02138, USA Instituut voor Sterrenkunde, Celestijnenlaan 200D, B-2001 Heverlee, Belgium e-mail: kkolenbe@cfa.harvard.edu
}

KASC WG13 (RR Lyrae working group)

http://astro.phys.au.dk/KASC/ 


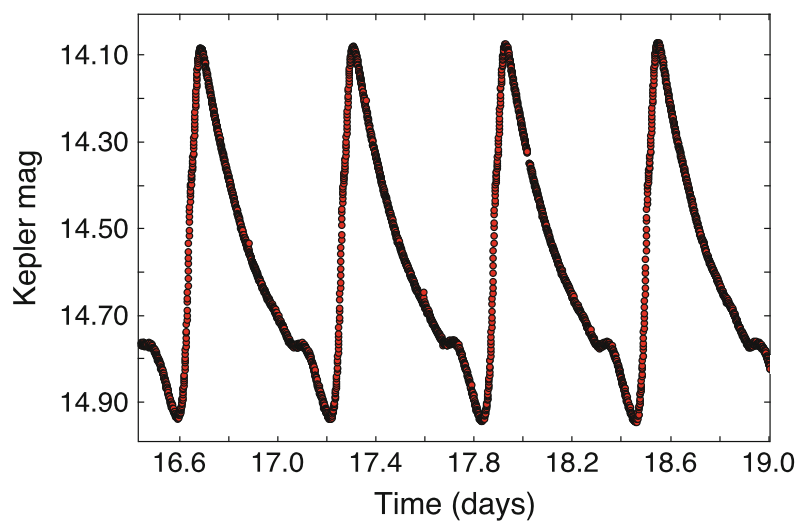

Fig. 1 Short cadence Kepler data of an RRab star

the bump closely before minimum light. Therefore, all of our targets are observed at least one quarter ( 3 months) in short cadence over the duration of the mission.

\section{Some Examples and Results}

\subsection{Time Series Analysis}

A first look at the Kepler data [9] already revealed that the incidence rate of the Blazhko effect in the Kepler field RR Lyraes is higher than the previously assumed $25-30 \%$ in Galactic populations. Moreover, additional frequencies, beyond the main pulsation mode, its harmonics and the Blazhko modulation components were detected for the first time. The half-integer frequencies associated with a period doubling effect were never before observed in RR Lyrae stars. A study based on the first quarters of all available data at the time [1] confirmed that about half of the RRab stars in our sample are Blazhko stars and modulation always occurs in both amplitude and phase. Furthermore, period doubling was found in several other Blazhko stars. In four stars, frequencies were found close to the first and/or second overtone modes, with amplitudes that seem to vary over the Blazhko cycle. Finally, two stars in our sample appear to pulsate in the fundamental and (lowamplitude) second overtone modes, thereby representing a new type of double-mode behavior $[1,13]$.

By a fortunate coincidence, RR Lyr itself, the prototype and by far the brightest star of the class, lies in the Kepler field. Thanks to a custom aperture devised by Steve Bryson (NASA Ames), the flux of this strongly saturated star could be recovered. RR Lyr offers a case study for observations of bright stars with Kepler [10]. 
One of the most challenging questions in analyzing Kepler data of a Blazhko star is how to correctly stitch together data from different quarters [4]. For modulated stars, it is indeed not trivial to disentangle whether variations between quarters are instrumental or stellar. Several interesting individual stars with variable Blazhko cycles are being studied in detail by working group members, e.g., $[4,6]$.

Interestingly, all four RRc variables known in the Kepler field thus far turned out to be multiperiodic, showing an additional period with a ratio to the main period of $\simeq 0.60-0.63$ (see [12]).

Non-modulated RR Lyrae stars allow us to study the stability of the pulsations of RR Lyrae stars and they provide a reference against which the Blazhko RR Lyrae stars can be compared [13]. From the Fourier spectra of these targets, $[\mathrm{Fe} / \mathrm{H}]$ values and other physical parameters can also be derived. From this, four of our target stars turn out to be particularly metal-rich.

\subsection{Theoretical Modelling}

When the observed periods of the non-modulated stars are compared with periods computed with the Warsaw non-linear convective pulsation code better agreement is achieved assuming pulsational luminosity $(L)$ and mass $(M)$ values rather than the (higher) evolutionary $L$ and $M$ values [13].

The detection of additional frequencies in many of our RR Lyrae targets has sparked modelling efforts to explain the observations. For the period doubling these modelling efforts are discussed extensively in these proceedings by Róbert Szabó [17]. These findings even led to a new model for the Blazhko effect in the framework of radial resonances [3]. Model calculations of RR Lyrae stars suggest that the bifurcation cascade, responsible for period doubling, may evolve to chaos in these dynamical systems [14]. The possibilities and occurrences of mutual resonances between the fundamental mode and multiple overtones was also investigated in hydrodynamical models [11].

A comparison between the modulation in the Kepler data of the prototype RR Lyr with hydrodynamical models [15], allowed to show some limitations of the Stothers model [16]. Finally, it still remains a riddle how to explain the newly detected frequencies in RRc stars. With our current knowledge of RR Lyrae pulsation models, they can only be explained in terms of non-radial modes.

\subsection{Ground-Based Follow-Up}

Multicolour photometry and spectroscopy has been gathered for the majority of our targets by several working group members. Besides applications for individual stars, this will allow us to better locate our sample stars in the HR diagram and to determine their metallicities. The high-resolution spectroscopy confirms the high 
metallicity of our targets that had emerged from the Fourier decomposition [13]. Time-resolved spectroscopic data would also allow a more literally in-depth study of the RR Lyrae variables.

\subsection{Additional Projects, and Stars!}

We know that there must be many more RR Lyrae stars in the Kepler field, and are actively searching for them. New pulsators can be found from the Kepler Full Frame Image Variability Catalog [7,8]. A few other RR Lyrae stars were already found through reclassification and some blended ones were found serendipitously (one even by an amateur through http://www.planethunters.org/!). We hope to significantly enlarge our sample of RR Lyrae stars before the end of the Kepler mission.

With the data from the Kepler mission major breakthroughs in our understanding of RR Lyrae stars have been and can/will be achieved. These data will provide material for thought and discovery for many years, possibly even decades, to come.

Acknowledgements We gratefully acknowledge the entire Kepler team, whose outstanding efforts have made these results possible. KK acknowledges Marie Curie Fellowship 2009-IOF255267.

\section{References}

1. Benkő, J.M., Kolenberg, K., Szabó, R., Kurtz, D.W., Bryson, S., et al.: Flavours of variability: 29 RR Lyrae stars observed with Kepler. MNRAS 409, 1585-1593 (2010)

2. Borucki, W.J., Koch, D., Basri, G., Batalha, N., Brown, T., et al.: Kepler Planet-detection mission: introduction and first results. Science 327, 977-980 (2010)

3. Buchler, J.R., Kolláth, Z.: On the Blazhko Effect in RR Lyrae Stars. ApJ 731, 24 (2011)

4. Çelik, L., Ekmekçi, F., Nemec, J., Kolenberg, K., Nemec, J., et al.: How to correctly stitch together data of a Blazhko Star? These proceedings (2011)

5. Gilliland, R.L., Brown, T.M., Christensen-Dalsgaard, J., Kjeldsen, H., Aerts, C., et al.: Kepler Asteroseismology program: introduction and first results. PASP 122, 131-143 (2010)

6. Guggenberger, E., Kolenberg, K., Nemec, J., Szabó, R., Benkő, J.: Changing Blazhko modulations: KIC6186029 in comparison with other cases of non-repetitive Blazhko cycles. These proceedings (2011)

7. Kinemuchi, K., Howell, S., Still, M., Szabó, R.: Selecting new pulsating stars from the Kepler full frame image variability catalog! These proceedings (2011)

8. Kinemuchi, K., et al.: in preparation

9. Kolenberg, K., Szabó, R., Kurtz, D.W., Gilliland, R.L., Christensen-Dalsgaard, J., et al.: First Kepler results on RR Lyrae stars. ApJL 713, L198-L203 (2010) 
10. Kolenberg, K., Bryson, S., Szabó, R., Kurtz, D.W., Smolec, R., et al.: Kepler photometry of the prototypical Blazhko star RR Lyr: an old friend seen in a new light. MNRAS 411, 878-890 (2011)

11. Molnár, L., Kolláth, Z., Szabó, R.: Uncovering hidden modes in RR Lyrae stars. These proceedings (2011)

12. Moskalik, P., Smolec, R., Kolenberg, K., Nemec, J., Kunder, A., Chadid, et al.: Discovery of peculiar double-mode pulsations and period doubling in Kepler c-type RR Lyrae stars. These proceedings (2011)

13. Nemec, J.M., Smolec, R., Benkö, J.M., Moskalik, P., Kolenberg, K., et al.: Fourier analysis of non-Blazhko ab-type RR Lyrae stars observed with the Kepler space telescope. MNRAS 417, 1022-1053 (2011)

14. Plachy, E., Kolláth, Z., Molnár, L.: Detection of chaos in RR Lyrae models. These proceedings (2011)

15. Smolec, R., Moskalik, P., Kolenberg, K., Bryson, S., Cote, M.T., Morris, R.L.: Variable turbulent convection as the cause of the Blazhko effect - testing the Stothers model. MNRAS 414, 2950-2964 (2011)

16. Stothers, R.B.: A new explanation of the Blazhko effect in RR Lyrae stars. ApJ 652, 643-649

17. Szabó, R., Kolláth, Z., Molnár, L., Kolenberg, K., Kurtz, D.W., WG13 members: Period doubling in Kepler RR Lyrae stars. These proceedings (2011) 